\title{
Using Skin Carotenoids to Assess Dietary Changes in Students After One Academic Year of Participating in the Shaping Healthy Choices Program
}

Key Words: skin carotenoids, resonance Raman spectroscopy, dietary assessment, nutrition education

Lori M Beccarelli, $\mathrm{PhD}^{1,2}$

3150G Meyer Hall

One Shields Ave.

Davis, CA 95616

530-752-3387

lmnguyen@ucdavis.edu

Rachel E Scherr*, PhD ${ }^{1,2}$

3149 Meyer Hall

One Shields Ave.

Davis, CA 95616

530-752-3817

rescherr@ucdavis.edu

Madan Dharmar, PhD ${ }^{3,4}$, MBBS

Education Building

4610 X Street, Suite 2301

Sacramento, CA 95817

916-734-2354

mdharmar@ucdavis.edu

Igor V Ermakov, $\mathrm{PhD}^{5}$

391 Chipeta Way

Salt Lake CIty, Utah 84108

801-583-3255

ermakov@physics.utah.edu

Werner Gellermann, $\mathrm{PhD}^{5}$

391 Chipeta Way

Salt Lake CIty, Utah 84108

801-583-3255

werner@physics.utah.edu

Lisa Jahns, $\mathrm{PhD}, \mathrm{RD}^{6}$

$24202^{\text {nd }}$ Ave. N

Grand Forks, ND 58202

701-795-8331

lisa.jahns@ars.usda.gov

Jessica D Linnell, $\mathrm{PhD}^{7}$

2204 Fourth Street

Tillamook, Oregon 97141

503-842-3433

Jessica.Linnell@oregonstate.edu 
Carl L Keen, PhD $1,8,9$

3135A Meyer Hall

One Shields Ave.

Davis, CA 95616

530-752-6331

clkeen@ucdavis.edu

Francene M Steinberg ${ }^{1,8}, \mathrm{PhD}, \mathrm{RD}$

3135B Meyer Hall

One Shields Ave.

Davis, CA 95616

530-752-0160

fmsteinberg@ucdavis.edu

Heather M Young PhD, RN, FAAN ${ }^{4}$

Education Building

4610 X Street

Sacramento, CA 95817

916-734-2145

heather.young@ucdmc.ucdavis.edu

Sheri Zidenberg-Cherr ${ }^{1,2,8}, \mathrm{PhD}$

3149 Meyer Hall

One Shields Ave.

Davis, CA 95616

530-752-3817

sazidenbergcherr@ucdavis.edu

${ }^{1}$ Department of Nutrition, University of California, Davis, Davis, CA

${ }^{2}$ Center for Nutrition in Schools, University of California, Davis, Davis, CA

${ }^{3}$ Department of Pediatrics, University of California Davis Health System, Sacramento, CA

${ }^{4}$ Betty Irene Moore School of Nursing, University of California Davis Health System,

Sacramento, CA

5Image Technologies Corporation, Salt Lake City, UT

6USDA-ARS Grand Forks Human Nutrition Research Center, Grand Forks, ND

${ }^{7}$ Family and Community Health, Extension Service - Tillamook and Lincoln Counties,

Oregon State University

8University of California Agriculture and Natural Resources, Davis, CA

${ }^{9}$ Department of Internal Medicine, University of California, Davis, Davis, CA

*corresponding author 
Objective: to determine whether 4th-grade students participating in the Shaping Healthy Choices Program (SHCP), a school-based nutrition intervention, change vegetable and carotenoid intake measured by skin carotenoids and dietary intake

Design: single group pre-test, post-test with a self-selected, convenience sample of students recruited at the school

Participants: 30 students had both dietary intake data and skin carotenoid measurements pre- and post-intervention.

Intervention(s): included garden-enhanced education, family and community partnerships, increased regionally procured produce in the lunchroom, and school-site wellness committees. Lasted one academic-year (nine months)

Main Outcome Measure(s): dietary intake of vegetables and carotenoids as measured by Block food frequency questionnaire and skin carotenoids as measured by resonance Raman spectroscopy.

Analysis: paired t-test, ANOVA and Pearson's correlation were used. Statistical significance set at $\alpha=0.05$

Results: Reported vegetable intake decreased by 0.4 cups $(\mathrm{P}=0.06)$. Reported carotenoid intake decreased by $1.5 \mathrm{mg}(\mathrm{P}=0.05)$ and skin carotenoids decreased by 2247.9 RRS intensity units $(\mathrm{P}=0.04)$. Change in reported intake correlated with change in skin carotenoids $(\mathrm{r}=0.43, \mathrm{P}=0.02)$

Conclusions and Implications: While the reported decrease in vegetable and carotenoid intake was unanticipated, the RRS measurements confirmed this. RRS data can help evaluate changes in fruit and vegetable intake. 


\section{Using Skin Carotenoids to Assess Dietary Changes in Students After One Academic Year}

\section{2 of Participating in the Shaping Healthy Choices Program}

\section{INTRODUCTION}

$4 \quad$ Most children in the U.S. are not meeting the Dietary Guidelines for Americans'

5 recommendations for fruit and vegetable intake. ${ }^{1}$ The Shaping Healthy Choices Program (SHCP)

6 was developed based on the Social Cognitive Theory ${ }^{2}$ and the Social Ecological Model ${ }^{3}$ to

7 encourage the consumption and enjoyment of fruits and vegetables. The SHCP is a multi-

8 component, school-based nutrition intervention that aims to improve dietary behaviors and

9 prevent childhood obesity by improving students' individual factors, as well as factors in the

10 home and school environments, while simultaneously creating a community-based support

11 system. ${ }^{4}$ In this study, changes in dietary intake of fruit and vegetable intake were evaluated

12 using two methods, reported dietary intake and skin carotenoids.

13 While numerous intervention studies aimed at increasing fruit and vegetable intakes in

14 children have been conducted, the assessment of changes in dietary behavior in children remains

15 a challenge. ${ }^{5-7}$ Reported dietary intake is often used in community-based interventions, however

16 it can be unreliable, and it is time and labor intensive. ${ }^{8-11}$ Skin carotenoid status measured by

17 resonance Raman spectroscopy (RRS) can be used as a biomarker to assess fruit and vegetable

18 intake because carotenoids are predominately found in fruits and vegetables and their presence in

19 the body can reflect cumulative dietary intakes of these foods. ${ }^{12-15}$ The RRS method is a quick,

20 noninvasive, and non-biased method to assess skin carotenoids and has previously been validated

21 in children. ${ }^{15}$ Skin carotenoid status using RRS has also been reported to change in response to

22 dietary intake, making it possible to use this methodology to detect changes in dietary

23 behavior. ${ }^{16,17}$ These reports, however, have largely been a result of feeding studies where there 
24 are substantial changes in carotenoid intake. Few studies to date have used RRS to measure

25 changes in usual dietary intake in children. Additionally, no studies have used this RRS

26 instrument to measure skin carotenoid changes in response to a multicomponent, school-based

27 nutrition program.

28 The objective of the current study was to determine whether students participating in the

29 SHCP changed their consumption of fruits, vegetables, and carotenoids measured by Food

30 Frequency Questionnaire (FFQ) and an RRS instrument that was developed for research

31 purposes (a research-grade RRS). A secondary objective was to determine whether the RRS

32 instrument could be useful to measure changes in fruit and vegetable intake after an intervention

33 lasting one academic year. It was hypothesized that students participating in the SHCP for one

34 academic year would increase consumption of vegetables and therefore have greater reported

35 intake of vegetables and carotenoids and greater skin carotenoid concentrations. Additionally, it

36 was hypothesized that an increased skin carotenoid intensity would be associated with a greater

37 carotenoid intake.

38 MATERIALS AND METHODS

39 Study Design: This study was a sub-study of the larger SHCP study based on available dietary

40 intake and skin carotenoid data. It was a single group pre-test, post-test design in which a self-

41 selected, convenience sample of students was assessed pre- and post-intervention.

42 Participants $(\mathbf{n}=\mathbf{3 0})$ : The students were recruited at the beginning of each academic year over

43 two years from $4^{\text {th }}$-grade classrooms in a Northern California elementary school participating in

44 the SHCP. ${ }^{4}$ The intervention was implemented twice, once over the course of the 2012-2013

45 academic year and repeated again in the 2013-2014 academic year. Exclusion criteria included

46 living in a smoking household or having illnesses or chronic conditions known to change 
47 carotenoid status. ${ }^{12,18}$ Under these criteria, no students were excluded from the study. The

48 University of California, Davis Institutional Review Board approved the study protocol. Parents

49 provided written informed consent and children provided oral assent.

50 Intervention: The SHCP intervention is described in detail elsewhere. ${ }^{4}$ Briefly, the SHCP aimed

51 to 1) increase nutrition knowledge and use of science processing skills; 2) promote availability,

52 consumption, and enjoyment of fruits and vegetables; 3 ) improve dietary patterns and encourage

53 physical activity; 4) foster positive changes in the school environment; and 5) facilitate

54 development of an infrastructure to sustain the program. Intervention activities included an

55 inquiry-based, garden-enhanced nutrition curriculum, Discovering Healthy Choices (DHC),

56 taught in the classroom; ${ }^{19}$ cooking demonstrations in the classroom using the Cooking $U p$

57 Healthy Choices curriculum; ${ }^{20}$ family newsletters sent home to parents; health fairs or health

58 screenings at the school; improvements to the school lunchroom including installing a salad bar

59 and increasing procurement of regional produce; and development of a school-site wellness

60 committee. In the 2012-2013 school year (August-May), the nutrition curriculum was delivered

61 by an SHCP educator. In the 2013-2014 school year (August-May), the intervention was

62 repeated with a new cohort of $4^{\text {th }}$-grade students and the teachers delivered the DHC curriculum

63 after participating in a comprehensive professional development program. Because the overall

64 results regarding dietary intake across both years were consistent, the students' data from both

65 years were combined.

66 Data Collection and Analysis: Pre-intervention data collection occurred before the intervention

67 activities began in August and post-intervention data collection occurred immediately after the

68 intervention's completion in May. Measurements included: 
69 Anthropometrics: height and weight were measured at the school site. ${ }^{4}$ The methods followed

70 guidelines published by the Centers for Disease Control and Prevention (CDC). Height was

71 measured using a transportable stadiometer (Seca 213, Chino, California) and body weight was

72 measured using an electronic scale (Seca 803b, Chino, California). Body Mass Index $\left(\mathrm{kg} / \mathrm{m}^{2}\right)$

73 was calculated and age and sex-specific BMI percentiles were derived. Classifications for

74 adiposity were based on those determined by the CDC and the Expert Committee on Childhood

75 and Adolescent Overweight and Obesity using the 2000 CDC growth charts. ${ }^{21-23}$ Under these $^{2}$

76 classifications, overweight was defined as at or above the $85^{\text {th }}$ percentile and obese was defined

77 as at or above the $95^{\text {th }}$ percentile of the sex-specific BMI-for-age growth chart. ${ }^{23}$

78 Demographics: race and ethnicity of the student were reported by the parents using a

79 demographic questionnaire that was sent home. ${ }^{4}$

80 Dietary intake: dietary intake was self-reported by students with the help of their parents at home

81 using the 2004 Block Food Frequency Questionnaire (FFQ) for Ages 8-17. Students were

82 provided detailed classroom instruction on completing the FFQ and were given standardized

83 bowls and a plate consistent with the diagram provided with the FFQ to use as a reference. ${ }^{4}$

84 Questionnaires were returned to the teachers for researchers to collect. Completed FFQs were

85 analyzed by NutritionQuest (Berkeley, CA). In the analysis report, $\alpha$-carotene, $\beta$-carotene, $\beta$ -

86 cryptoxanthin, lutein, and lycopene were quantified. Total dietary carotenoids were calculated by

87 taking the sum of the individual carotenoids reported by the FFQ analysis. Total vegetables

88 (minus white potatoes) was calculated by taking the difference between total vegetables and

89 white potatoes reported by the FFQ analysis report.

90 Skin carotenoids: In the 2012-2013 school year, pre-intervention total skin carotenoids were

91 assessed in November/December using RRS at a health fair event organized by SHCP 
92 researchers at the school site. Student attendance at the health fair was optional but required for

93 participation in the evaluation. In May 2013, a similar health fair event was hosted and students

94 volunteered to return for post-intervention measurements. In the 2013-2014 school year, skin

95 carotenoids were assessed in November/December using RRS and measurements were

96 conducted during class time. Post-intervention skin carotenoid data was collected May 2014

97 during class time. The instrument and method used in this study were developed for research

98 purposes by members of this group and have been validated in diverse populations and details

99 are reported elsewhere. ${ }^{12,13,15,24-26}$ Due to the overlap on the absorption spectra for all

100 carotenoids, individual carotenoid intensity is unavailable using this instrument.

101 Statistical Methods: Inclusion in this sub-study of students was based on available data and

102 those without post-intervention data were excluded. Means and standard deviations were

103 calculated for continuous variables and distributions were examined for normality. All variables

104 followed a nearly normal distribution and were not transformed. Percentages were calculated for

105 categorical variables. Change was measured by taking the difference between pre-and post-

106 intervention measurements. Paired t-tests were used to determine differences in skin carotenoid

107 intensity, BMI percentile, and dietary consumption of fruit, vegetables, total carotenoids, and

108 energy between pre-and post-intervention time points. Student's t-tests were also used to

109 determine whether there were differences between those who had only pre-intervention data and

110 both pre/post-intervention data. Variables considered for this analysis were race/ethnicity,

111 gender, age, RRS intensity, and reported dietary intake of vegetables and carotenoids. Pearson's

112 correlation coefficients were used to determine the relationship between change in skin

113 carotenoid intensity and change in reported dietary intake of carotenoids, change in reported

114 energy intake, and change in BMI percentile. Because students who typically consume low 
115 amounts of fruits and vegetables may show a different pattern of change following an

116 intervention, ${ }^{27}$ a post-hoc sub-analysis of the larger SHCP study was conducted using paired t-

117 tests to evaluate differences between pre-and post-intervention in students who reported

118 consuming less than one serving of vegetables/day pre-intervention. Stata 13 (StataCorp, College

119 Station, Texas) was used for all statistical analyses. Statistical significance was determined using

120 an $\alpha=0.05$.

\section{RESULTS}

122 Of the 249 students enrolled in the larger SHCP study who were eligible to participate in

123 this study over the two years, 30 students (12\%) who had both RRS and FFQ data (FFQ+RRS

124 group, $\mathrm{n}=30$ ) pre-and-post intervention were self-selected for this sub-study. Baseline

125 characteristics of the participants are presented in Table 1. In this group, skin carotenoid

126 measurements decreased by 2247.9 Intensity Units (IU) post-intervention ( $\mathrm{P}=0.04$, Table 2 ) and

127 dietary intake of carotenoids significantly decreased by $1.5 \mathrm{mg}$ post-intervention $(\mathrm{P}=0.05$, Table

128 2). There was no significant difference regarding reported total fruit or vegetable consumption

129 (Table 2).

130 Dietary intake of carotenoids correlated with skin carotenoids pre-intervention $(\mathrm{r}=0.46$,

$131 \mathrm{P}=0.001$, data not shown) and post-intervention ( $\mathrm{r}=0.52, \mathrm{P}=0.001$, data not shown). The change

132 in dietary carotenoids correlated with the change in skin carotenoid intensity $(\mathrm{r}=0.43, \mathrm{P}=0.02$,

133 Table 3). There was no correlation between the change in skin carotenoids with the change in

134 reported energy intake and change in BMI percentile (Table 3).

135 In post-hoc analyses of students in the larger SHCP study with pre-and post-intervention

136 FFQ data, there were 39 out of 93 students (42\%) who reported consuming less than one serving 
137 of vegetables per day at baseline. After participation in the intervention, these students reported a

138 significant increase of 0.4 cups of vegetables $(\mathrm{P}=0.01$, Table 2$)$ and no change in fruit intake.

\section{DISCUSSION}

140 The decrease in reported dietary intake of vegetables and skin carotenoids was

141 unanticipated given that the SHCP intervention aimed to increase consumption of fruits and

142 vegetables. No change in fruit intake was anticipated as youth this age consume near to adequate

143 amounts of fruit. ${ }^{29}$ Other multi-component and school-based interventions that aim to increase

144 fruit and vegetable intake have also reported little or no change in vegetable intake. ${ }^{28-30}$ One

145 explanation for the decrease in skin carotenoids in this study may be the differences in offering

146 of fruits and vegetables in the school at the beginning of the intervention (November/December)

147 compared to the end (May). Vegetables offered in the lunchroom pre-intervention included

148 autumn vegetables, such as sweet potatoes, squash, and butternut squash, which are more

149 carotenoid-rich, compared to vegetables offered in the spring, such as celery, cucumbers, and

150 snap peas. Lower skin carotenoids were also observed in studies that measured skin carotenoids

151 over time. ${ }^{31}$ There was no correlation between the change in carotenoid intake and change in

152 energy intake, so it is unlikely that the decrease in reported carotenoid intake is related to a

153 decrease in total overall energy intake. Studies have indicated that there is an inverse relationship

154 between skin carotenoids and BMI. ${ }^{12,13,15}$ However, there was no relationship between change in

155 skin carotenoids and change in BMI percentile in this study population, so any changes in BMI

156 do not appear to be influencing change in skin carotenoids.

157 The decrease in reported dietary intake of carotenoids was confirmed by the skin

158 carotenoid measurements, indicating that the RRS instrument was able to detect this small

159 decrease. This suggests that this subset of participants was consuming fewer carotenoid-rich 
160 fruits and vegetables following the intervention. Regardless of the reason for a decreased

161 reported carotenoid intake, the change in reported carotenoid intake correlated with the change in

162 skin carotenoids. Thus, the RRS instrument was able to distinguish a change in skin carotenoids

163 reflected by a small change $(1.5 \mathrm{mg})$ in reported intake. These results confirm that this method is

164 an objective way to measure the carotenoid status of children and can be used to help evaluate

165 changes in fruit and vegetable intake.

166 While there was an overall decrease in reported vegetable consumption, this was not the

167 case for students who reported consuming less than one serving of vegetables per day pre-

168 intervention. In post-hoc analyses of the original SHCP study, these students significantly

169 increased consumption of vegetables post-intervention. This phenomenon of low consumers

170 consuming more vegetables post-intervention compared to high consumers has also been

171 observed in other studies that aim to increase intake of fruits and vegetables. ${ }^{27,32}$ This is $^{2}$

172 noteworthy as this suggests that those students who are consuming very little vegetables at

173 baseline are likely to benefit most from the intervention, therefore it is this group of students that

174 should be targeted by interventions that aim to improve dietary behavior.

175 In this study, vegetable intake was examined because the SHCP intervention focused on

176 consuming more nutrient dense vegetables with less focus on fruit since children are already

177 close to meeting the dietary recommendations for fruit. ${ }^{33}$ Vegetable intakes were examined with

178 and without white potatoes, because while they are the most highly consumed vegetable, most

179 Americans consume white potatoes in a form that adds calories (such as French fries and potato

180 chips) while being less nutrient dense, with less carotenoid concentration, compared to other

181 vegetables. ${ }^{34}$ This was true of this study population; white potatoes were consumed mostly as

182 French fries and tater tots (data not shown). There was a slight difference in total vegetable 
183 intake calculated with and without white potatoes, though this difference was not statistically

184 significant. However, by examining vegetable intake with and without white potatoes, it can be

185 determined whether increases or decreases in intake in total vegetables are due to changes in

186 white potato consumption.

187 A strength of this study is that it used an objective, validated, research-grade evaluation

188 tool to measure changes in skin carotenoids in children participating in a school-based, nutrition

189 intervention. The instrument used in this study was extensively validated in controlled feeding

190 studies and over extended periods of time against plasma and skin biopsies. Previous studies in

191 children have used commercialized RRS technologies to measure changes in skin carotenoids

192 resulting from provision of high-carotenoid foods or incentivizing children to consume high-

193 carotenoid foods. ${ }^{17,27}$ Though the commercialized instrument is based on the research-grade

194 instrument, there is no published data regarding the similarities or differences between the two

195 instruments. While most studies have shown that RRS can measure increases in fruit and

196 vegetable consumption, results from this study demonstrate that the tool can also reflect

197 decreases in consumption, critical as a useful tool must be able to detect any change. This study

198 shows that even small changes in dietary intake resulting from behavior change within an

199 academic school year can be measured. This suggests that this tool can be used reliably to

200 evaluate other school-based nutrition interventions that aim to change fruit and vegetable intake

201 behavior. While the hypothesized outcome of increased vegetable intake was not achieved

202 through evaluating mean intakes in this study population, it is still important to share these

203 results. Too often, negative or unexpected outcomes are not published, yet these results are still

204 important in order to maintain an unbiased body of scientific knowledge. ${ }^{35}$ Additionally, many of 
205 these studies may potentially yield interesting results. It is therefore important to evaluate the 206 variation in the data, which this current study has done.

207 One limitation of this study is the lack of sun exposure data, which has been reported to 208 affect skin carotenoid status. ${ }^{17,18,31}$ It is suggested that carotenoids have photoprotective effects

209 and can be oxidized by sunlight, therefore increased sun exposure can diminish skin carotenoid

210 status. ${ }^{12}$ It is possible that participants in this study spent more time in the sun at follow-up given

211 the warmer weather of the spring months in Northern California, and this could help explain a

212 decrease in skin carotenoids. Additionally, skin carotenoids were measured at two different times

213 of the year, when fruit and vegetable availability and intake may be different. The FFQ used in

214 this study is not specifically designed to measure carotenoid intake and is restricted by a fixed

215 food list and portion size options, which may affect the reporting of carotenoid intake.

216 Regardless of this, reported dietary carotenoids correlated with skin carotenoid measurements,

217 suggesting that the FFQ assessed carotenoid intake with some accuracy. Despite the small

218 sample size, there was enough power for the instrument to detect changes, suggesting that this

219 tool is sensitive enough to detect small changes in carotenoid intake.

\section{IMPLICATIONS FOR RESEARCH AND PRACTICE}

221 In regards to the changes in dietary intake in the larger SHCP intervention, more research

222 is required to elucidate reasons for the decrease in reported vegetable intake. However, in post-

223 hoc analysis of the SHCP, looking at those who consumed less than one serving of vegetables

224 per day, a reported increase in vegetable intake was observed. This implies that the SHCP is a

225 promising intervention that may have a greater benefit to those who are furthest from meeting

226 dietary recommendations of vegetables. This can provide further insight into planning future

227 nutrition education studies and help studies target the most vulnerable populations. This also 
emphasizes the need to not just simply focus on means, but to separate the data in a meaningful

229 that way might yield results that may otherwise be overlooked.

230 For the students in the RRS+FFQ group, it is evident that the decrease in reported

231 carotenoid intake was reflected by the decrease in skin carotenoid intensity, implying that small

232 changes in dietary intake of carotenoids is related to a measurable change in skin carotenoids.

233 Biomarker data can help strengthen evaluations of future nutrition intervention studies and

234 educational programs aimed at increasing fruit and vegetable intakes in children. RRS is a quick

235 method that requires little effort on behalf of the participants and can be used to provide an

236 objective measure to help evaluate changes in dietary intake. Future studies using RRS should

237 consider sun exposure, timing of measurements, and larger sample sizes.

\section{REFERENCES:}

240 1. Krebs-Smith SM, Guenther PM, Subar AF, Kirkpatrick SI, Dodd KW. Americans do not

2. Bandura A. Social foundations of thought and action: A social cognitive theory. Englewood Cliffs, NJ, US: Prentice-Hall, Inc; 1986.

3. Dietary guidelines for Americans 2015-2020. Washington, DC: United States Department of Health and Human Services;2015.

4. Scherr RE, Linnell JD, Smith MH, et al. The Shaping Healthy Choices Program: design and implementation methodologies for a multicomponent, school-based nutrition education intervention. J Nutr Ed Behav. 2014;46(6):e13-21.

5. Cullen KW, Watson K, Zakeri I. Relative reliability and validity of the Block Kids Questionnaire among youth aged 10 to 17 years. J Am Diet Assoc. 2008;108(5):862-866.

6. Lim SS, Gold A, Gaillard PR, Wey A, Reicks M. Validation of 2 Brief Fruit and Vegetable Assessment Instruments Among Third-Grade Students. J Nutr Ed Behav. 2015;47(5):446-451 e441.

7. Tabacchi G, Amodio E, Di Pasquale M, Bianco A, Jemni M, Mammina C. Validation and reproducibility of dietary assessment methods in adolescents: a systematic literature review. Public Health Nutr. 2014;17(12):2700-2714.

8. Livingstone MB, Robson PJ, Wallace JM. Issues in dietary intake assessment of children and adolescents. Br J Nutr. 2004;92 Suppl 2:S213-222.

9. Sharman SJ, Skouteris H, Powell MB, Watson B. Factors Related to the Accuracy of Self-Reported Dietary Intake of Children Aged 6 to 12 Years Elicited with Interviews: A Systematic Review. J Acad Nutr Diet. 2015. 
10. Burrows TL, Martin RJ, Collins CE. A systematic review of the validity of dietary assessment methods in children when compared with the method of doubly labeled water. $J$ Am Diet Assoc. 2010;110(10):1501-1510.

11. Shim JS, Oh K, Kim HC. Dietary assessment methods in epidemiologic studies. Epidemiol Health. 2014;36:e2014009.

12. Mayne ST, Cartmel B, Scarmo S, Jahns L, Ermakov IV, Gellermann W. Resonance Raman spectroscopic evaluation of skin carotenoids as a biomarker of carotenoid status for human studies. Arch Biochem Biophys. 2013;539(2):163-170.

13. Scarmo S, Henebery K, Peracchio H, et al. Skin carotenoid status measured by resonance Raman spectroscopy as a biomarker of fruit and vegetable intake in preschool children. Eur J Clin Nutr. 2012;66(5):555-560.

14. Aguilar SS, Wengreen HJ, Lefevre M, Madden GJ, Gast J. Skin carotenoids: a biomarker of fruit and vegetable intake in children. J Acad Nutr Diet. 2014;114(8):1174-1180.

15. Nguyen LM, Scherr RE, Linnell JD, et al. Evaluating the relationship between plasma and skin carotenoids and reported dietary intake in elementary school children to assess fruit and vegetable intake. Arch Biochem Biophy. 2015;572:73-80.

16. Jahns L, Johnson LK, Mayne ST, et al. Skin and plasma carotenoid response to a provided intervention diet high in vegetables and fruit: uptake and depletion kinetics. Am J Clin Nut. 2014;100(3):930-937.

17. Aguilar SS, Wengreen HJ, Dew J. Skin Carotenoid Response to a High-Carotenoid Juice in Children: A Randomized Clinical Trial. J Acad Nutr Diet. 2015;115(11):8.

18. Darvin ME, Patzelt A, Knorr F, Blume-Peytavi U, Sterry W, Lademann J. One-year study on the variation of carotenoid antioxidant substances in living human skin: influence of dietary supplementation and stress factors. J Biomed Opt. 2008;13(4):044028.

19. Linnell JD, Zidenberg-Cherr S, Briggs M, et al. Using a Systematic Approach and Theoretical Framework to Design a Curriculum for the Shaping Healthy Choices Program. J Nutr Ed Behav. 2016;48(1):60-69 e61.

20. Center for Nutrition in Schools. Cooking Up Healthy Choices. 2015; http://cns.ucdavis.edu/programs/shcp/cooking.html. Accessed 28 April, 2016.

21. Barlow SE. Expert committee recommendations regarding the prevention, assessment, and treatment of child and adolescent overweight and obesity: summary report. Pediatrics. 2007;120 Suppl 4:S164-192.

22. Kuczmarski RJ, Ogden CL, Guo SS, et al. 2000 CDC Growth Charts for the United States: methods and development. Vital and health statistics. Series 11, Data from the national health survey. 2002(246):1-190.

23. Prevention CDC. Defining Childhood Obesity. http://www.cdc.gov/obesity/childhood/defining.html. Accessed December 9, 2015.

24. Ermakov IV, Gellermann W. Validation model for Raman based skin carotenoid detection. Arch Biochem Biophys. 2010;504(1):40-49.

25. Ermakov IV, Sharifzadeh M, Ermakova M, Gellermann W. Resonance Raman detection of carotenoid antioxidants in living human tissue. J Biomed Opt. 2005;10(6):064028.

26. Gellermann W, Zidichouski J, Smidt A, Bernstein PS. Raman detection of carotenoids in human tissue. In: Klaus Kraemer LP, Helmut Sies, and Ute Obermüller-Jevic, ed. Carotenoids and Retinoids: AOCS Publishing; 2005. 
27. Wengreen HJ, Madden GJ, Aguilar SS, Smits RR, Jones BA. Incentivizing children's fruit and vegetable consumption: results of a United States pilot study of the Food Dudes Program. J Nutr Educ Behav. 2013;45(1):54-59.

28. Evans CE, Christian MS, Cleghorn CL, Greenwood DC, Cade JE. Systematic review and meta-analysis of school-based interventions to improve daily fruit and vegetable intake in children aged 5 to 12 y. Am J Clin Nutr. 2012;96(4):889-901.

29. Prelip M, Kinsler J, Thai CL, Erausquin JT, Slusser W. Evaluation of a school-based multicomponent nutrition education program to improve young children's fruit and vegetable consumption. J Nutr Educ Behav. 2012;44(4):310-318.

30. Evans CE, Ransley JK, Christian MS, Greenwood DC, Thomas JD, Cade JE. A clusterrandomised controlled trial of a school-based fruit and vegetable intervention: Project Tomato. Public Health Nutr. 2013;16(6):1073-1081.

31. Scarmo S, Cartmel B, Lin H, et al. Single v. multiple measures of skin carotenoids by resonance Raman spectroscopy as a biomarker of usual carotenoid status. Br J Clin Nutr. 2013;110(5):911-917.

32. Horne PJ, Tapper K, Lowe CF, Hardman CA, Jackson MC, Woolner J. Increasing children's fruit and vegetable consumption: a peer-modelling and rewards-based intervention. Eur J Clin Nutr. 2004;58(12):1649-1660.

33. Kim SA, Moore LV, Galuska D, et al. Vital signs: fruit and vegetable intake among children - United States, 2003-2010. MMWR. Morbidity and mortality weekly report. 2014;63(31):671-676.

34. Lin BH, Wendt M, Guthrie JF. Impact on energy, sodium and dietary fibre intakes of vegetables prepared at home and away from home in the U.S.A. Public Health Nutr. 2013;16(11):1937-1943.

35. Matosin N, Frank E, Engel M, Lum JS, Newell KA. Negativity towards negative results: a discussion of the disconnect between scientific worth and scientific culture. Disease Models \& Mechanisms. 2014;7(2):171-173. 
336 Table 1: Participant Characteristics in the RRS+FFQ group $(n=30)$ at Baseline.

\begin{tabular}{|c|c|}
\hline & RRS+FFQ group \\
\hline Average Age \pm SD (years) & $9.9 \pm 0.6$ \\
\hline $\begin{array}{c}\text { Sex, } \mathrm{n}(\%) \\
\text { Females } \\
\text { Males }\end{array}$ & $\begin{array}{l}21(70 \%) \\
9(30 \%)\end{array}$ \\
\hline Mean BMI percentile \pm SD & $74.5 \pm 25.3$ \\
\hline $\begin{array}{l}\text { BMl-for-age Percentile } \\
\text { Normal weight }\left(5^{\text {th }}-<85^{\text {th }} \text { percentile }\right) \\
\text { Overweight }\left(85^{\text {th }}-<95^{\text {th }} \text { percentile }\right) \\
\text { Obese }\left(\geq 95^{\text {th }} \text { percentile }\right)\end{array}$ & $\begin{array}{l}13 \\
12 \\
5\end{array}$ \\
\hline $\begin{array}{l}\text { Race/Ethnicity, } \mathrm{n}(\%) \\
\text { African American/Black, not of } \\
\quad \text { Hispanic origin } \\
\text { Asian/Pacific Islander } \\
\text { Caucasian/White, not Hispanic origin } \\
\text { Latino/Hispanic (Mexican-American, } \\
\quad \text { Puerto Rican, Cuban) } \\
\text { Other } \\
\text { Multiple reported } \\
\text { Not reported }\end{array}$ & $\begin{array}{l}3(10 \%) \\
11(37 \%) \\
8(27 \%) \\
1(3 \%)\end{array}$ \\
\hline
\end{tabular}


338 Table 2: Comparison of Pre-and Post-Intervention Means \pm SD of Skin Carotenoid Intensity, 339 Reported Dietary Intakes, and BMI Percentile for the RRS+FFQ Group $(n=30)$ and comparison of 340 Pre-and Post-Intervention Means \pm SD of Reported Dietary Intakes and BMI Percentile of 341 Participants Who Reported Less Than One Serving of Vegetables Per Day Pre-intervention $342 \quad(n=39)$.

\begin{tabular}{|c|c|c|c|c|}
\hline & Pre-Intervention & Post-Intervention & Mean difference & $P$-Value \\
\hline \multicolumn{5}{|l|}{ RRS+FFQ group $(n=30)$} \\
\hline & \multicolumn{2}{|c|}{$\leftarrow$ Mean \pm SD $\rightarrow$} & & \\
\hline $\begin{array}{l}\text { Skin carotenoids, intensity } \\
\text { units }\end{array}$ & $\begin{array}{c}32580.4 \pm \\
11629.1 \\
\end{array}$ & $\begin{array}{c}30332.4 \pm \\
10329.7\end{array}$ & $-2247.9 \pm 5671.3$ & $0.04 *$ \\
\hline Dietary carotenoids, mg & $7.7 \pm 5.0$ & $6.2 \pm 4.1$ & $-1.5 \pm 4.0$ & $0.05 *$ \\
\hline Vegetables, cups & $1.2 \pm 1.1$ & $0.9 \pm 0.5$ & $0.4 \pm 1.0$ & 0.06 \\
\hline $\begin{array}{l}\text { Vegetables (excluding } \\
\text { white potatoes), cups }\end{array}$ & $1.1 \pm 0.9$ & $0.8 \pm 0.1$ & $0.3 \pm 0.9$ & 0.12 \\
\hline $\begin{array}{l}\text { Leafy green vegetables, } \\
\text { cups }\end{array}$ & $0.3 \pm 0.4$ & $0.3 \pm 0.3$ & $0.1 \pm 0.4$ & 0.38 \\
\hline Orange vegetables, cups & $0.1 \pm 0.2$ & $0.1 \pm 0.1$ & $0.1 \pm 0.2$ & 0.17 \\
\hline Fruit, cups & $1.5 \pm 1.0$ & $1.8 \pm 1.3$ & $0.2 \pm 1.5$ & 0.43 \\
\hline Total energy, calories & $1437.3 \pm 888.2$ & $1256.9 \pm 545.6$ & $-180.3 \pm 831.4$ & 0.25 \\
\hline BMI percentile & $74.5 \pm 25.3$ & $71.3 \pm 25.7$ & $-1.8 \pm 10.1$ & 0.10 \\
\hline \multicolumn{5}{|c|}{$<1$ serving vegetables/day $(n=39)$} \\
\hline & \multicolumn{2}{|c|}{$\leftarrow$ Mean \pm SD $\rightarrow$} & & \\
\hline Dietary carotenoids, mg & $4.3 \pm 21.7$ & $6.1 \pm 4.1$ & $1.9 \pm 1.0$ & 0.06 \\
\hline Vegetables, cups & $0.5 \pm 0.2$ & $0.9 \pm 0.8$ & $0.4 \pm 0.9$ & $0.01 *$ \\
\hline $\begin{array}{l}\text { Vegetables (excl. white } \\
\text { potatoes), cups }\end{array}$ & $0.4 \pm 0.2$ & $0.8 \pm 0.5$ & $0.4 \pm 0.8$ & $<0.01 *$ \\
\hline $\begin{array}{l}\text { Leafy green vegetables, } \\
\text { cups }\end{array}$ & $0.1 \pm 0.1$ & $0.2 \pm 0.1$ & $0.1 \pm 0.3$ & $0.01 *$ \\
\hline Orange vegetables, cups & $0.0 \pm 0.0$ & $0.1 \pm 0.2$ & $0.1 \pm 0.2$ & $0.01^{*}$ \\
\hline Fruit, cups & $1.4 \pm 1.0$ & $1.7 \pm 1.2$ & $0.3 \pm 1.6$ & 0.20 \\
\hline Total energy, calories & $1072.0 \pm 503.5$ & $1362.6 \pm 778.0$ & $290.6 \pm 892.5$ & $0.05 *$ \\
\hline BMI percentile & $74.7 \pm 26.1$ & $63.2 \pm 30.3$ & $-11.5 \pm 20.0$ & $0.002 *$ \\
\hline
\end{tabular}
$\mathrm{FFQ}=$ Food Frequency Questionnaire

344 RRS = Resonance Raman Spectroscopy

345 *Statistically significant using an $\alpha=0.05$ 
347 Table 3: Pearson's Correlation Between Change in Skin Carotenoid Intensity and Change in 348 Reported Intake of Carotenoids, Change in Total Energy Intake, and Change in BMI Percentile in 349 the RRS+FFQ Group $(n=30)$.

\begin{tabular}{|c|c|c|}
\hline $\begin{array}{c}\text { Change in Skin } \\
\text { Carotenoid Intensity vs: }\end{array}$ & Correlation coefficient & P-Value \\
\hline $\begin{array}{c}\text { Change in Reported } \\
\text { Intake of Carotenoids }\end{array}$ & 0.43 & $0.02^{*}$ \\
\hline $\begin{array}{c}\text { Change in Total Energy } \\
\text { Intake }\end{array}$ & 0.28 & 0.13 \\
\hline $\begin{array}{c}\text { Change in BMI } \\
\text { Percentile }\end{array}$ & 0.03 & 0.87 \\
\hline
\end{tabular}

$350 \quad F F Q=$ Food Frequency Questionnaire

351 RRS = Resonance Raman Spectroscopy

352 *Statistically significant using an $\alpha=0.05$ 